\title{
Chinese Stroke Association guidelines for clinical management of cerebrovascular disorders: executive summary and 2019 update of clinical management of stroke rehabilitation
}

To cite: Zhang T, Zhao J, Li X, et al. Chinese Stroke Association guidelines for clinical management of cerebrovascular disorders: executive summary and 2019 update of clinical management of stroke rehabilitation. Stroke \& Vascular Neurology 2020;5: e000321. doi:10.1136/svn-2019-000321

- Additional material is published online only. To view please visit the journal online (http://dx.doi.org/10.1136/svn2019-000321).

Received 15 January 2020 Revised 19 May 2020 Accepted 27 May 2020 Published Online First 28 June 2020

Check for updates

(c) Author(s) (or their employer(s)) 2020. Re-use permitted under CC BY-NC. No commercial re-use. See rights and permissions. Published by BMJ.

For numbered affiliations see end of article.

Correspondence to Dr Tong Zhang; ztcrrc@126.com

\section{ABSTRACT}

Stroke is the third leading cause of long-term disability. Effective stroke rehabilitation should be provided to patients with a moderate or severe stroke so as to achieve functional recovery. Also, stroke rehabilitation is moving towards more intense therapy models that incorporate technologies such as robotics and computer games. Evidence-based guidelines are useful for promoting the best possible clinical practices. This guideline summarises the modes of stroke rehabilitation in China-three-stage rehabilitation network, rehabilitation of stroke dysfunction, training for activities of daily living, stroke complications and rehabilitation care. The aim of this guideline was to provide a synopsis of best clinical practices in the stroke rehabilitation of adult patients in China.

There are about 2 million new stroke cases in China each year, and $70 \%$ to $80 \%$ of patients who had a stroke cannot live independently due to disability. Despite advances in modern medicine, medications and medical technology, stroke diseases impose a substantial mortality and morbidity risk to the individual with increased economic burden to the society. As such, there is an urgent need to develop an optimal stroke disease management plan, incorporating a comprehensive stroke rehabilitation programme. What is more, stroke rehabilitation is moving towards more intense therapy models that incorporate technologies such as robotics and computer games.

The purpose of the Stroke Rehabilitation Guideline is to provide scientific evidence and basis for the implementation and evaluation of rehabilitation therapy, standardise the rehabilitation treatment post-stroke, help the medical institutions follow the evidence-based rehabilitation methodology and improve the rehabilitation results.

\section{MODE OF STROKE REHABILITATION-THREE-} STAGE REHABILITATION NETWORK

A large number of studies have shown that three-stage rehabilitation after stroke (figure 1) can improve motor function and reduce complications. This implies early rehabilitation in stroke units and neurological wards, subacute rehabilitation in medical wards or rehabilitation hospital followed by long-term rehabilitation at a rehabilitation centre or the community or at home.

\section{Recommendations}

1. Three-stage rehabilitation network should be promoted, so that patients can continue lifelong rehabilitation (Grade I recommendation, Level A evidence).

2. Stroke rehabilitation units should be established in general hospitals and rehabilitation centres (Grade I recommendation, Level A evidence).

3. Patients with chronic stroke should be recommended to transfer to the tertiary rehabilitation institution for subsequent rehabilitation with unified rehabilitation programme (Grade I recommendation, Level B evidence).

\section{Early rehabilitation}

Early rehabilitation and rehabilitation teams

Early rehabilitation is generally performed within 1 month after stroke onset. The Stroke Unit should provide rehabilitation, which includes training for improvement of extremity dysfunction, language use, cognition and so on.

Early rehabilitation intervention time and rehabilitation intensity

In 2011, the Chinese stroke rehabilitation guidelines recommended that patients who 


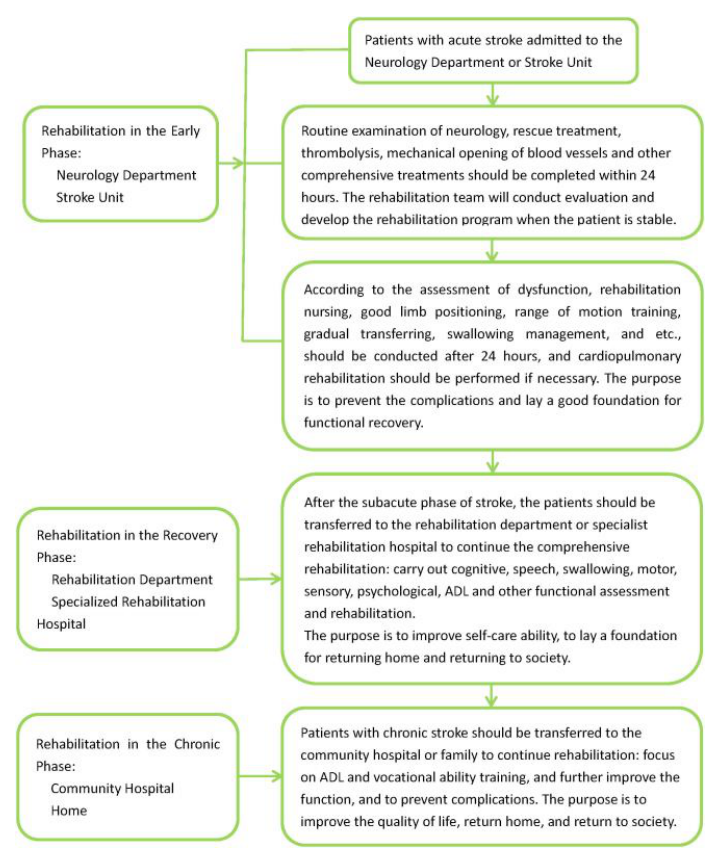

Figure 1 Flowchart of three-stage rehabilitation after stroke. $A D L$, activities of daily living.

had a stroke should be rehabilitated as soon as stable (stable vital signs, no signs of progression). ${ }^{1}$ Rehabilitation that starts within 2 weeks following stroke can achieve better outcome. ${ }^{2}$ A Very Early Rehabilitation Trial (AVERT) explored the effectiveness and safety of superearly activities within 24 hours after stroke onset. The results showed that the super-early activity programme could reduce the benefit at 3 months. ${ }^{3}$

\section{Recommendations}

1. It is recommended that patients with acute stroke are admitted to a Stroke Unit of the General Hospital. These patients should immediately undergo a comprehensive assessment, while early rehabilitation should be provided by the rehabilitation team when patients reach stability (Grade I recommendation, Level A evidence).

2. When stable, patients with acute stroke should be evaluated for individualised and comprehensive rehabilitation (Grade I recommendation, Level A evidence).

3. It is reasonable to apply standard and effective scale for the rehabilitation assessment before developing individualised programme and rehabilitation (Grade IIa, Level B evidence).

4. Patients who had a stroke should rehabilitate as soon as possible when clinically stable (vital signs stable, symptoms and signs no longer progress) through the step-by-step training method (Grade I recommendation, Level A evidence).

5. Mobilisation within 24 hours after stroke onset is not recommended because it may reduce the possibility for a good outcome at 3 months (Grade III recommendation, Level B evidence).
6. It is reasonable for patients who had a mild and moderate stroke to do bedside rehabilitation and early rehabilitation away from bed 24 hours after stroke onset. Rehabilitation should be conducted in a step-by-step manner, under supervision if necessary (Grade IIa recommendation, Level A evidence).

7. The intensity of rehabilitation should be individualised, and the patient's physical strength, endurance and cardiopulmonary function should be taken into account. The rehabilitation is reasonable to last at least 45 min per day if possible at the early stage, after which it is beneficial to appropriately increase the intensity (Grade IIa recommendation, Level B evidence).

8. Goal-oriented instructions may be considered to lead to significant increases in the rate of exercise repetitions, as well as an increase in the intensity of practice in stroke rehabilitation (Grade IIb recommendation, Level B evidence).

\section{Rehabilitation in the recovery period}

Rehabilitation in the recovery period generally refers to the rehabilitation from 1 month to 6 months after stroke onset.

\section{Recommendations}

1. Patients who had a stroke in the recovery stage should be admitted to the rehabilitation department in a general hospital and rehabilitation hospital (Grade I recommendation, Level B evidence).

2. It is recommended to apply standard and effective scales (Grade I recommendation, Level B evidence).

3. Stroke rehabilitation during the recovery stage should be comprehensive, providing the foundation for a return to home and society (Grade I recommendation, Level C evidence).

\section{Chronic rehabilitation \\ Recommendations}

1. It is reasonable for community hospitals to provide secondary rehabilitation (Grade IIa recommendation, Level B evidence).

2. After rehabilitation in general hospital and rehabilitation hospital, patients should seek the community rehabilitation for lifelong rehabilitation (Grade I recommendation, Level A evidence).

3. The evidence on the effectiveness of remote stroke rehabilitation is still insufficient (Grade IIa recommendation, Level B evidence).

4. Patients who had a stroke may also benefit from community rehabilitation after discharge from the hospital (Grade I recommendation, Level A evidence).

5. It is reasonable for family members to participate in patient self-management programmes in communitybased rehabilitation (Grade IIa, Level B evidence).

6. General-disciplinary rehabilitation management, collaborative health management or group management in community rehabilitation are recommended (Grade I recommendation, Level B evidence). 
7. Activities of daily living (ADL) can be significantly improved in the three-stage rehabilitation and is recommended as reinforcement plan (Grade I recommendation, Level A evidence).

8. Constraint-induced movement therapy is recommended to improve ADL (Grade I recommendation, Level A evidence).

9. Wii-based exercise therapy is reasonable to improve the motor function of the affected upper limb and ADL (Grade IIa recommendation, Level B evidence).

10. Transcranial direct current stimulation combined with virtual reality may be beneficial in improving patients' quality of life (Grade IIb recommendation, Level B evidence).

11. A comprehensive and multidisciplinary team is reasonable to provide occupational rehabilitation (Grade IIa recommendation, Level B evidence).

12. Vagus nerve stimulation paired with rehabilitation is acceptably safe and feasible. It is reasonable to improve upper limb motor function after chronic ischaemic stroke (Grade IIa recommendation, Level B evidence).

\section{REHABILITATION OF STROKE DYSFUNCTION}

\section{Rehabilitation of dyskinesia}

\section{Recommendations}

1. A patient who had a stroke should be kept on the bed in a normal limb position. Lateral position on the af fected side is recommended (Grade I recommenda tion, Level C evidence).

2. It is reasonable that normal limb position can effectively reduce limb spasticity and improve the rehabilitation outcomes (Grade IIa recommendation, Level B evidence).

3. Transfer training should be performed with patients who had a stroke on-bed with the help of attendant or rehabilitation therapist as soon as possible (Grade I recommendation, Level B evidence).

4. Range of motion training is recommended for patients who had a stroke on-bed, and mechanical injury of the affected limbs should be avoided (Grade I recommendation, Level B evidence).

5. Transferring and range of motion training is reasonable to implement as early as possible and could prevent complications, such as hypostatic pneumonia, deep venous thrombosis (DVT) and bedsores (Grade IIa recommendation, Level B evidence).

6. When clinically stable, patients with acute stroke with paralysis should get out of the bed as soon as possible, and start training standing and walking (Grade I recommendation, Level B evidence).

7. In the acute stroke phase, the muscle strength training for the paralysed limbs should be emphasised (Grade I recommendation, Level B evidence).

8. Functional electrical stimulation and electromyography biofeedback for the corresponding muscles, combined with conventional rehabilitation, can improve the muscle strength and the function of paralysed limbs (Grade I recommendation, Level B evidence).

9. It is reasonable for patients who had a stroke with hemiplegia to train standing and walking actively to regain basic walking ability as soon as possible (Grade IIa recommendation, Level B evidence).

10. Analysis of the hemiplegic gait with an integrative gait analysis system is an effective way to make an elaborate walking programme and improve the quality of walking (Grade I recommendation, Level B evidence).

11. Lower-limb robots, weight-supporting devices and orthoses is reasonable to assist with walking recovery (Grade IIa recommendation, Level B evidence).

12. The Modified Ashworth Scale and Modified Tardieu Scale are recommended for the evaluation of spasticity (Grade I recommendation, Level B evidence).

13. Although the electromyography operation is time consuming and complicated, it may lead to some advantages (Grade IIb recommendation, Level B evidence).

14. The principle of post-stroke spasticity treatment is to improve functional ability (Grade I recommendation, Level C evidence).

15. Normal limb position, passive stretching, range of motion training and Chinese medical massages are recommended to alleviate spasticity (Class I recommendation, Level $\mathrm{C}$ evidence).

16. Neuromuscular electrical stimulation (NMES) and local muscle vibration therapy are recommended (Grade I recommendation, Level B evidence).

17. Oral medications, including eperisone, baclofen and tizanidine, are recommended, and local botulinum toxin injection is recommended for local muscle spasm after stroke (Grade I recommendation, Level B evidence).

18. Combined transcranial direct current stimulation, repetitive transcranial magnetic stimulation and transcutaneous electrical nerve stimulation (TENS) with conventional physiotherapy is reasonable to relieve spasticity (Grade IIa recommendation, Level B evidence).

19. It is reasonable that acupuncture used selectively in combination with clinical practice in spasticity after stroke (Grade IIa recommendation, Class B evidence).

\section{Rehabilitation of sensory dysfunction}

Recommendations

1. Sensory impairment, including somatosensory, visual and hearing, is recommended for assessment of patients who had a stroke (Grade I recommendation, Level C evidence).

2. It is reasonable to use various sensory stimuli for rehabilitation in patients with sensory disturbance after stroke (Grade IIa recommendation, Level B evidence). 


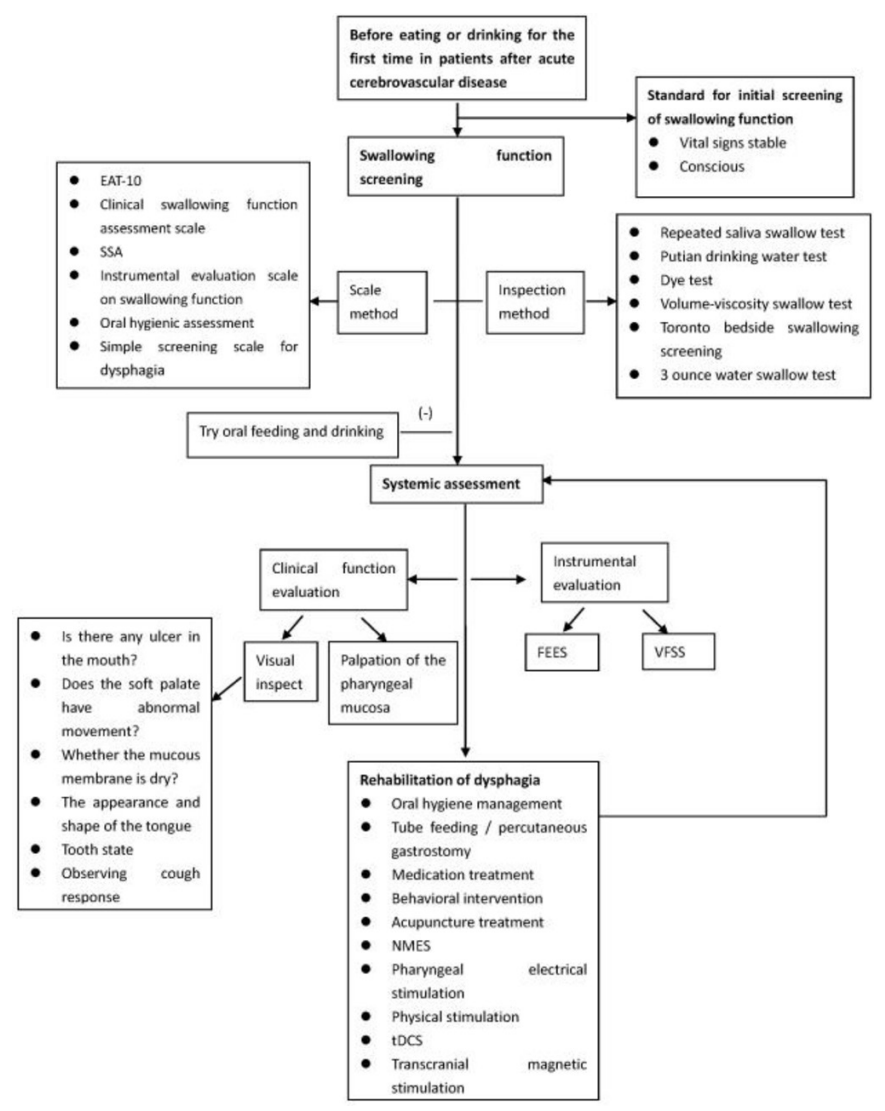

Figure 2 Flowchart of rehabilitation of swallowing dysfunction after stroke.

3. It is reasonable to use TENS in patients with sensory impairment after stroke (Grade IIa recommendation, Level B evidence).

4. Virtual reality may be considered to improve sensory perception function (Grade IIb recommendation, Level C evidence).

5. Compensatory saccade training is reasonable to improve functional deficits after visual field loss and improve functional ADL. However, this approach cannot reduce visual impairment effectively (Grade IIa recommendation, Level B evidence).

6. Prisms is reasonable to be used as a complementary therapy to help patients compensate for visual field defects (Grade IIa recommendation, Level B evidence).

7. Virtual reality is reasonable to be considered to improve the visual-spatial function (Grade IIa recommendation, Level B evidence).

\section{Rehabilitation of swallowing dysfunction}

Overview

Dysphagia after stroke affects $42 \%$ to $67 \%$ of patients within 3 days after the onset of stroke, where $50 \%$ tend to have aspiration (including occult aspiration) and $1 / 3$ pneumonia. ${ }^{4}$ Figure 2 shows the assessment and rehabilitation flowchart of the swallowing function in patients who had a stroke. Early screening for swallowing function after stroke is recommended; however, currently, there are no randomised controlled studies on the optimal timing of screening for swallowing function. ${ }^{5}$

\section{Screening for dysphagia}

Commonly used assessments include the Eating Assessment Tool-10 (EAT-10), the Clinical Swallowing Function Assessment Form, the Standardised Swallowing Assessment (SSA) and so on. The inspection methods include repeated saliva swallowing test, Watian drinking water test, dye test and so on. ${ }^{6-11}$

\section{Recommendations}

1. All patients with acute stroke are recommended to undergo screening for the swallowing function before oral feeding and drinking water by a speech therapist or other trained healthcare people (Grade I recommendation, Level A evidence).

2. It is reasonable for patients with suspected aspiration to be further examined with flexible endoscopic examination of swallowing (FEES) or/and videofluoroscopic swallowing study (VFSS) (Grade IIa recommendation, Level B evidence).

3. FEES or/and VFSS, which are supplementary to each other, is reasonable to be used for instrumental examination of dysphagia (Grade IIa recommendation, Level B evidence).

4. All patients with dysphagia is reasonable to be evaluated for nutrition and water supply within 48 hours (Grade IIa recommendation, Level B evidence).

5. Behavioural intervention is considered a part of swallowing treatment; oral hygiene management can reduce the risk of post-stroke aspiration pneumonia (Grade I recommendation, Level B evidence).

6. Acupuncture treatment is reasonable to be considered as a potentially adjuvant treatment for dysphagia (Grade IIa recommendation, Level B evidence).

7. Currently, there is no sufficient evidence on beneficial effects of medication, NMES, pharyngeal electrical stimulation, physical stimulation, transcranial direct current stimulation (tDCS) and transcranial magnetic stimulation (Grade IIb recommendation, Level B evidence).

8. Patients who had a stroke who could not eat safely and effectively should start enteral nutrition (tube feeding) within 7 days after stroke (Grade I recommendation, Level A evidence).

9. Patients who had a stroke who could not eat safely and effectively for more than 4 weeks were suggested percutaneous gastrostomy tube insertions (Grade IIa recommendation, Level B evidence).

\section{Dysarthria}

\section{Overview}

Dysarthria can individually or simultaneously affect any speech production subsystem, including the respiratory, larynx, pharynx and oral pronunciation subsystems. ${ }^{12} 13$ 


\section{Recommendations}

1. Communication assessments should include interviews, dialogues, observations, standardised tests, assessment of speech, language use and reading (Class IIa recommendation, Level B evidence).

2. Interventions for motor speech impairment should be individualised, including targeted behavioural techniques and strategies (Class I recommendation, Level B evidence).

3. Auxiliary communication devices and treatments for speech therapy should be used as complementary means (Class I recommendation, Level C evidence).

4. Environmental adjustments, including audience education, is reasonable to be used to improve communication (Class IIa recommendation, Level C evidence).

5. It is reasonable to use activities that promote social participation and improve psychosocial health (Class IIa recommendation, Level C evidence).

\section{Aphasia}

\section{Assessment of aphasia}

Screening can be performed for patients with post-stroke communication impairment before total language assessments. Boston diagnostic aphasia examination (BDAE) Chinese version and western aphasia battery mainly focus on the diagnosis and classification of aphasia. Most parts of BDAE (Chinese version) have good inter-inspector reliability and retest reliability, while in some small parts, such as execution instructions and rhythm sub-tests, the re-test correlation is low due to difficulty. Remote rehabilitation is becoming a recognised alternative to face-to-face communication assessment for patients with communication disorders; however, it requires adequate technical support. ${ }^{14-16}$

\section{Rehabilitation of aphasia}

\section{Recommendations}

1. Communication assessments should include interviews, dialogues, observations, standardised tests or non-standardised projects for assessment of speech, language, cognition, language use, reading and writing; identifying advantages and weaknesses of communication; and identifying useful compensatory strategies (Class I recommendation, Level B evidence).

2. Remote rehabilitation is reasonable to employ when the face-to-face assessment is impossible or impractical (Class IIa recommendation, Level A evidence).

3. It is reasonable to use individualised interventions for the treatment of cognitive-communication disorders (Class IIa recommendation, Level B evidence).

4. Aphasia rehabilitation for aphasia patients is recommended (Class I recommendation, Level A evidence).

5. Intensive treatment is likely to be necessary, but there is no consensus on the optimal quantity, strength, distribution or duration (Class IIa recommendation, Level B evidence).
6. Computer therapy is reasonable to be used as an addition to speech and language therapy (SLT) (Class IIa recommendation, Level B evidence).

7. Group therapy for aphasia is reasonable at each stage of treatment, including the application of community aphasia groups (Class IIa recommendation, Level B evidence).

8. Brain stimulation may be considered as an adjunct experimental therapy to behavioural SLT, thus regular use is not recommended (Class IIb recommendation, Level B evidence).

9. Acupuncture is reasonable for aphasia treatment (Class IIa recommendation, Level B evidence).

10. Use of music is reasonable for aphasia rehabilitation (Class IIa recommendation, Level B evidence).

\section{Rehabilitation of cognitive impairment}

Introduction

Existing studies have shown that post-stroke cognitive impairment after stroke within 3 months ranges from $6 \%$ to $27 \% .{ }^{1718}$

\section{Assessment of cognitive impairment}

Minimum Mental State Examination (MMSE) and Montreal Cognitive Assessment (MoCA) ${ }^{19}{ }^{20}$ could be used to screen post-stroke cognitive impairment. Previous studies have found that MMSE has a ceiling effect and low sensitivity, ${ }^{21}{ }^{22}$ while MoCA is more sensitive with lower specificity. ${ }^{23} 24$

\section{Recommendations}

1. Screening for cognitive impairment is recommend ed for all patients who had a stroke. When screening suggested cognitive impairment, a more detailed netı ropsychological assessment should be used (Class I rec ommendation, Level B evidence).

2. Rich environment is recommended to increase participation in cognitive activities (Class I recommendation, Level A evidence).

3. It is reasonable to use cognitive rehabilitation to improve attention, memory and executive function (Class IIa recommendation, Level B evidence).

4. Cognitive training strategies include practice, compensation and adaptation techniques (Class IIa recommendation, Level B evidence).

5. Compensation strategy can be used to improve memory function (Class IIa recommendation, Level B evidence).

6. Virtual reality training is reasonable for speech, visual and spatial learning (Class IIa recommendation, Level B evidence).

7. Exercise is reasonable as an adjunct to improve cognitive and memory after stroke (Class IIa recommendation, Level C evidence).

8. tDCS for improvement of complex attention (working memory) is still being researched (Class IIa recommendation, Level B evidence). 
9. Strategic training or posture training for limb apraxia should be considered (Class IIa recommendation, Level B evidence).

10. Task practice with or without motor imaginary training for limb disability should be considered (Class IIa recommendation, Level $\mathrm{C}$ evidence).

11. Computerised treatment of self-administration of oral apraxia may be considered (Class IIa recommendation, Level C evidence).

12. It is reasonable to repeat visual interventions such as shuttle lens adaptation, visual scanning training, visual stimulation, virtual reality, physical activity, mental imagery, prism adaptation and neck vibration to improve neglect symptoms (Class IIa recommendation, Level B evidence).

13. Repetitive transcranial magnetic stimulation and tDCS may be considered for improvement of neglected symptoms (Class IIa recommendation, Level B evidence).

14. The role of donepezil, nimodipine and memantine in the treatment of cognitive impairment after stroke is not fully established (Class IIa recommendation, Level B evidence).

\section{Impaired cardiopulmonary function}

Assessment of cardiopulmonary function

Patients who had a stroke often show varying degrees of impaired cardiopulmonary function, peak levels of $\mathrm{VO}_{2}$ drop to $8-22 \mathrm{~mL} / \mathrm{kg} \times \min$ (about $53 \%$ of the normal value of age and gender matching population). ${ }^{25}$ The peak level of $\mathrm{VO}_{2}$ necessary for independent living is $15-18 \mathrm{~mL} / \mathrm{kg} \times \mathrm{min}$, so cardiopulmonary exercise capacity after stroke is a key factor affecting the quality of life and functional recovery. ${ }^{26}$

\section{Recommendations}

1. Physical activity level and activities related to daily life after stroke should be assessed (Class IIa recommendation, Level C evidence).

2. Asymptomatic function assessment and cardiopulmonary function assessment should be performed as soon as possible (Class I recommendation, Level B evidence).

3. Post-stroke pneumonia should be first treated with antibiotics. It is reasonable to enhance the management of respiratory secretions (Class IIa recommendation, Level B evidence).

4. It is necessary to guide the body position, food nutrition and traits during feeding so as to improve nutritional support and the immunity of patients (Class I recommendation, Level B evidence).

5. Individualised rehabilitation programme is recommended for stroke survivors to improve cardiopulmonary function (Class I recommendation, Level B evidence).

6. Patients with stroke in bed should receive regular exercise rehabilitation training. Patients with stroke with sufficient strength in lower limb muscles are rec- ommended to strengthen cardiovascular adaptability training (Class IIa recommendation, Level B evidence).

7. Patients with severe stroke are advised to do strengthening exercises bedside and respiratory rehabilitation (Class IIa recommendation, Level B evidence).

8. Blood oxygen partial pressure, oxygen saturation, vital capacity and $1 \mathrm{~s}$ forced respiration after stroke can be used as indicators of lung function (Class IIa recommendation, Level B evidence).

\section{Rehabilitation of mental disorder}

Post-stroke depression (PSD)

PSD is one of the common complications of a stroke, occurring at the rate of about $9 \%$ to $34 \% .{ }^{27}{ }^{28}$ PSD slows the recovery of the patient's impairment, reduces the quality of life and even increases the patient's mortality rate. $^{29}$

\section{Assessment of PSD}

While there is no consensus on the best screening method, ${ }^{30}$ the scales commonly used for PSD include the Hospital Anxiety and Depression Scale, and the Hamilton Depression Scale. For patients with aphasia and cognitive impairment, the current effective depression rating scales are mainly Aphasic Depression Rating Scale (ADRS) and Visual Analog Mood Scale.

\section{Recommendations}

1. In the absence of contraindications, patients with PSD should receive antidepressant treatment (Class I recommendation, Level B evidence).

2. It is recommended to use a structural depression scale, such as the Patient Health Questionnaire-2 for routine PSD screening (Class I recommendation, Level B evidence).

3. ADRS is reasonable for psychological assessment of patients with PSD. A single assessment should be completed within 24 hours. It is recommended to be assessed once a week (Class IIa recommendation, Level C evidence).

4. Serotonin reuptake inhibitors can be used to treat PSD (Class I recommendation, Level A evidence).

5. For patients with emotional distress or pseudobulbar affect, selective serotonin reuptake inhibitor or dextromethorphan/quinidine should be considered (Class IIa recommendation, Level A evidence).

6. It is reasonable to provide a professional mental or psychiatrist consultation for stroke survivors with a persistent affective disorder or disability deterioration (Class IIa recommendation, Level C evidence).

7. Patient education, counselling services and social support may be considered as part of PSD treatment (Class IIb recommendation, Level B evidence).

8. It is recommended to use mindfulness decompression and mindfulness cognitive therapy for PSD (Class I recommendation, Level A evidence). 
9. Combined drugs and transcranial electromagnetic stimulation, hyperbaric oxygen therapy, exercise therapy and music therapy should be considered for the treatment of PSD (Class IIa recommendation, Level B evidence).

10. It is reasonable to use the Baker Anxiety Scale to assess post-stroke anxiety (Class IIa recommendation, Level C evidence).

11. It is recommended to use serotonin reuptake inhibitors to treat post-stroke anxiety (Class I recommendation, Level A evidence).

12. It is recommended to use mindfulness decompression and mindfulness cognitive therapy for PSD (Class I recommendation, Level A evidence).

\section{ADL, SOCIAL PARTICIPATION BARRIERS AND VOCATIONAL TRAINING}

\section{$A D L$ and social engagement disorder}

Recommendations

1. It is recommended to use Barthel Index and Modified Barthel Index to assess ADL in patients with stroke (Class I recommendation, Level B evidence).

2. Because of high reliability and sensitivity, functional independence measure is recommended (Class I recommendation, Level A evidence).

3. Early rehabilitation, occupational therapy, constraintinduced movement therapy, virtual reality rehabilitation training, functional electrical stimulation, repetitive transcranial magnetic stimulation and other methods are recommended for improvement of ADL (Class I recommendation, Level A evidence).

\section{Vocational training and guidance}

In a study with 8 years of follow-up, the risk of unemployment in young patients who had a stroke increased two to three times. ${ }^{31}$

\section{Recommendations}

Semi-structured interviews should be used to understand influencing factors and needs of patients to return to work; assessment and training in the workplace can increase the probability of returning to work (Class I recommendation, Level B evidence).

\section{COMPLICATIONS}

\section{Skin damage}

Recommendations

1. In patients who had a stroke, maintaining sufficient nutrient intake, water supply and dynamic position management can effectively reduce the risk of skin damage (Class I recommendation, Level A evidence).

2. Special air bed is recommended to use to avoid skin friction (Class I recommendation, Level B evidence).

\section{Contracture}

A prospective cohort study found that the probability of multiple joint contractures has been estimated to be $12 \%$ to $28 \% .^{32}$ In a broad retrospective analysis of 328 stroke survivors, the health score of the patients with spasm were significantly lower than those in non-spasm patients $(\mathrm{p}<0.05){ }^{33}$

\section{Recommendations}

1. Active exercise training is reasonable to provide with patients who have a high risk of contracture (Class IIa recommendation, Level B evidence).

2. Functional electrical stimulation therapy and extracorporeal shock wave therapy is reasonable for relieving contracture (Class IIb recommendation, Level B evidence).

3. Oral anti-muscle tension drugs and local muscles for botulinum toxin targeted injection therapy is reasonable for long-term hypertonic contracture (Class IIa recommendation, Level B evidence).

4. When conservative treatment is ineffective, surgical lysis or orthopaedic adjuvant therapy may be considered (Class IIa recommendation, Level B evidence).

\section{Deep venous thrombosis}

\section{Recommendations}

1. Patients with DVT should receive regular anticoagulant therapy (Class I recommendation, Level A evidence).

2. Intermittent inflation and intermittently applying pressure to the calf muscles and blood vessels is reasonable to stimulate blood flow (Class IIa recommendation, Level A evidence).

3. Compression stockings should not be performed to treat and prevent the development of DVT and pulmonary embolism (Class III recommendation, Level B evidence).

\section{Intestinal and bladder incontinence}

Age, aphasia, impaired urinary awareness and dyskinesia are the leading causes of urinary incontinence. ${ }^{34}$ Pizzi $e t$ $a l$, who monitored the urodynamic pattern in 84 patients with post-stroke urinary incontinence, suggested that post-stroke urinary incontinence was associated with different changes in urodynamic patterns, and each urodynamic model may require different treatment strategies. ${ }^{35}$ Ersoz et al studied the bladder emptying method at various stages after stroke and suggested that urinary retention should be assessed by bladder scan or intermittent catheterisation volume after urination. ${ }^{36}$

\section{Recommendations}

1. It is recommended to evaluate bladder function of patients with acute stroke (Class I recommendation, Level B evidence).

2. Regular urination and pelvic floor muscle training should be considered for urinary incontinence in patients who had a stroke after discharge (Class IIa recommendation, Level B evidence).

3. Intestinal function assessment should include stool hardness, frequency and time of defecation before the stroke, and a history of bowel treatment (Class IIa recommendation, Level C evidence). 


\section{Hemiplegic shoulder pain}

Recommendations

1. It is recommended to educate patients and their families about post-stroke prevention of shoulder pain and shoulder care (Class I recommendation, Level C evidence).

2. Clinical evaluation of hemiplegia shoulder pain includes musculoskeletal assessment, spasm assessment, shoulder subluxation and local sensory disturbance examination (Class I recommendation, Level C evidence).

3. Botulinum toxin injection can effectively alleviate the pain caused (Class IIa recommendation, Level A evidence).

4. For patients with subluxation of the shoulder joint, position fixation and supportive devices and shoulder straps should be considered (Class IIa recommendation, Level B evidence).

5. Ultrasound can be used as a diagnostic tool for shoulder soft-tissue injury. Acupuncture, NMES, suprascapular nerve block, and subacromial or shoulder joint injection of corticosteroids can be used as an adjuvant treatment for hemiplegia (Class IIb recommendation, Level B evidence).

\section{Shoulder-hand syndrome (SHS)}

The main clinical symptoms of SHS are pain, paresthesia, vascular dysfunction, oedema, abnormal sweating and nutritional disorders. ${ }^{37} 38$

TENS can stimulate spinal cord fibres, thus inhibiting the activity of small-diameter pain-transmitting fibres, and having a certain therapeutic effect on SHS. ${ }^{39}$

\section{Recommendations}

1. In patients with SHS, it is reasonable to moderately raise the affected limb in co-ordination with passive activities. Combination with neuromuscular electrical stimulation is more effective (Class IIa recommendation, Level B evidence).

2. For patients with obvious swelling of the hand, shortterm steroid therapy should be considered (Class IIa recommendation, Level B evidence).

3. External compression device should be considered to reduce swelling of the extremity of the limb (Class IIa recommendation, Level B evidence).

\section{Central post-stroke pain (CPSP)}

CPSP is mainly caused by damage to the ventral basal complex of the thalamus. ${ }^{40}$ Some researchers have also demonstrated a causal relationship between CPSP and neuronal activity in the ventral posterolateral nucleus of the thalamus. ${ }^{41}$

\section{Recommendations}

1. Individualised choice of CPSP treatment should be tailored according to patients' needs, treatment response and adverse reactions; multidisciplinary pain management combined with medication may also be effective (Class I recommendation, Level C evidence).
2. Amitriptyline and lamotrigine are reasonably considered as first-line treatments, while pregabalin, gabapentin, carbamazepine or phenytoin are considered as second-line treatment (Class IIa recommendation, Level B evidence).

\section{Osteoporosis after stroke}

Patients who had a stroke often have a deficiency of vitamin $\mathrm{D},{ }^{42} 43$ which leads to secondary hyperthyroidism.

\section{Recommendations}

It is reasonable to develop individualised aerobic exercise and strength training for patients who had a stroke, which can reduce the risk of osteoporosis and fracture after stroke (Class IIa recommendation, Level B evidence).

\section{REHABILITATION CARE}

Early implementation of acute stroke rehabilitation care can reduce or prevent further complications.

\section{Recommendations}

It is recommended to implement comprehensive rehabilitation care interventions during rehabilitation to meet the specific needs of patients with stroke (Class I recommendation, Level B evidence).

\section{Health education and psychological care Recommendations}

1. It is recommended to provide health education for patients with mild stroke to improve their awareness of changes in their health status, initiative and confidence in the rehabilitation, and improve the overall rehabilitation quality (Class I recommendation, Level C evidence).

2. It is reasonable to conduct inspiring interviews and personalised education for patients so as to define individual risk factors, which may be beneficial for the long-term control of stroke risk factors (Class IIa recommendation, Level B evidence).

\section{Respiratory management}

\section{Recommendations}

In patients who had a stroke with declined respiratory function and intrapulmonary infection, the bedside respiratory management and pulmonary function rehabilitation should be strengthened (Class IIa recommendation, Level B evidence).

\section{Fall prevention}

Studies have shown that about $40 \%$ of patients who had a stroke have experienced a fall within 6 months after stroke. ${ }^{44}$ The fall prevention programme should be implemented in the early intervention of stroke, and it is important to timely identify the high-risk patients. It is necessary to carry out proper inpatient facility environmental transformation and safety education. ${ }^{45}{ }^{46}$ Disposal and prevention of falls should be addressed by the multidisciplinary team. 


\section{Recommendations}

1. During the hospitalisation period, each patient with a stroke should be assessed for fall risks and provided with an individualised fall prevention programme. Patients who are in the community are recommended to participate in balance training, such as Tai Chi, to prevent falls (Class I recommendation, Level B evidence).

2. Patients with poor balance, low self-confidence, fear of falling or risks of falling should receive balance function training (Class IIa recommendation, Level C evidence).

3. An annual assessment of the risk of falls should be conducted to improve the life and home environment in patients who had a stroke, and to reduce the accident (Class IIa recommendation, Level B evidence).

\section{Position management and oropharyngeal care}

\section{Recommendations}

1. It is recommended to perform oropharyngeal care and health assessment in patients who had a stroke, as well as a routine assessment of swallowing ability before oral intake (Class I recommendation, Level B evidence).

2. It is reasonable to raise the head of the bed by $20^{\circ}-45^{\circ}$ to reduce reflux aspiration caused by improper position (Class IIa recommendation, Level C evidence).

\section{Author affiliations}

${ }^{1}$ Department of Neurorehabilitation, China Rehabilitation Research Center, Beijing, China

${ }^{2}$ Department of Rehabilitation, Nanjing First Hospital, Nanjing, Jiangsu, China ${ }^{3}$ Department of Rehabilitation, Huashan Hospital Fudan University, Shanghai, China ${ }^{4}$ Department of Neurorehabilitation, Inner Mongolia Baotou City Central Hospital, Baotou, Inner Mongolia, China

${ }^{5}$ Department of Neurorehabilitation, Sichuan University West China Hospital, Chengdu, Sichuan, China

Collaborators Chinese Stroke Association Stroke Council Guideline Writing Committee. Chairmen: Yongjun Wang, yongjunwang@ncrcnd.org.cn, Department of Neurology, Beijing Tiantan Hospital, Capital Medical University, Beijing, China; Jizong Zhao, zhaojz205@163.com / zhaojz@public.bta.net.cn, Department of Neurosurgery, Beijing Tiantan Hospital, Capital Medical University, Beijing, China. Vice-Chairmen: Qiang Dong, dong_qiang@fudan.edu.cn, Department of Neurology, Huashan Hospital, Fudan University, Shanghai, China; Anding Xu, tli@jnu.edu. cn, Department of Neurology and Stroke Center, the First Affiliated Hospital, Jinan University, Guangzhou, China. Members of Academic Committee: Kangning Chen, ckn_640827@126.com' Department of Neurology, The Southwest Hospital, the First Affiliated Hospital of Third Military Medical University, Chongqing, China; Junbo Ge, ge.junbo@zs-hospital.sh.cn' Shanghai Institute of Cardiovascular Diseases, Department of Cardiology, Zhongshan Hospital, Fudan University, Shanghai, China; Li Guo, guoli6@163.com, Department of Neurology, The Second Hospital of Hebei Medical University, Shijiazhuang, China; Li He, heli2003new@126.com, Department of Neurology, West China Hospital, Sichuan University, Chengdu, China; Bo Hu, hubo@hust.edu.cn, Department of Neurology, Union Hospital, Tongji Medical College, Huazhong University of Science and Technology (HUST), Wuhan, China; Yong Huo, huoyong@263.net.cn' Department of Cardiology, Peking University First Hospital, Beijing, China; Linong Ji, jiln@bjmu.edu.cn, Department of Endocrinology and Metabolism, Peking University People's Hospital, Medicine at Peking University, Beijing, China; Xunming Ji, robertjixm@hotmail.com / jixunming@vip.163.com, Department of Neurosurgery, Xuanwu Hospital, Capital University of Medicine, Beijing, China; Tielin Li, tielin2013@126.com / tielin.li@tom.com, Zhujiang Hospital of Southern Medical University, Guangzhou, China; Liping Liu, lipingsister@ gmail.com, Department of Neurology, Beijing Tiantan Hospital, Capital Medical University, Beijing, China; Benyan Luo, luobenyan@zju.edu.cn, Department of
Neurology, $1^{\text {st }}$ Affiliated Hospital of Zhejiang University, Hangzhou, China; Zhongrong Miao, zhongrongm@163.com, Department of Interventional Neuroradiology, Beijing Tiantan Hospital, Capital Medical University, Beijing, China; Xiaoyuan Niu, niuxiaoyuan1958@163.com, Department of Neurology, First Hospital of Shanxi Medical University, Taiyuan, China; Bin Peng, pengbin3@hotmail.com; Department of Neurology, Peking Union Medical College Hospital, Peking Union Medical College and Chinese Academy of Medical Sciences, Beijing, China; Dingfeng Su, dfsu@ smmu.edu.cn, Department of Pharmacology, the Second Military Medical University (SMMU), Shanghai, China; Beisha Tang, bstang7398@163.com, Department of Neurology, Xiangya Hospital, Central South University, Changsha, China; Chen Wang, wangchen-tr2002@163.com, Beijing Tiantan Hospital, Capital Medical University, Beijing, China; Ning Wang, nwang900@yahoo.com, Department of Neurology and Institute of Neurology, First Affiliated Hospital of Fujian Medical University, Fuzhou, China; Shuo Wang, captain9858@vip.sina.com, Department of Neurosurgery, Beijing Tiantan Hospital, Capital Medical University, Beijing, China; Wei Wang, wwang@vip.126.com / wwang@tjh.tjmu.edu.cn, Tongji Hospital, Tongji Medical College, Huazhong University of Science and Technology, Wuhan, China; Xin Wang, wang.xin@zs-hospital.sh.cn, Department of Neurology, Zhongshan Hospital, Fudan University, Shanghai, China; Yilong Wang, yilong528@aliyun.com, Department of Neurology, Beijing Tiantan Hospital, Capital Medical University, Beijing, China; Shizheng Wu, wushizheng2005@hotmail.com, Qinghai Province People's Hospital, Xining, China; Peng Xie, xiepeng@cqmu.edu.cn, Chongqing Medical University (CQMU), Chongqing, China; Yuming Xu, 13903711125@126. com / xym13903711125@126.com, Department of Neurology, the First Affiliated Hospital of Zhengzhou University, Zhengzhou, China; Yun Xu, xuyun20042001@ aliyun.com, Department of Neurology, Drum Tower Hospital, Medical School of Nanjing University, Nanjing, China; Yi Yang, doctoryangyi@163.com / doctor_ yangyi@hotmail.com, Department of Neurology, the First Hospital of Jilin University, Changchun, China; Jinsheng Zeng, zengjs@pub.guangzhou.gd.cn, Department of Neurology and Stroke Center, the First Affiliated Hospital of Sun Yat-Sen University, Guangdong, China; Chaodong Zhang, scdzhang@163.com, The First affiliated Hospital of China Medical University, Shenyang, China; Tong Zhang, zt61611@ sohu.com, Capital Medical University School of Rehabilitation Medicine, China Rehabilitation Research Center, Beijing, China; Zhuo Zhang, zzhu0005@gmail. com, Beijing Anzhen Hospital, Capital Medical University, Beijing, China; Gang Zhao, zhaogang@fmmu.edu.cn, Department of Neurology, Xijing Hospital, The 4th Military Medical University, Xi'an, China; Xingquan Zhao, zxq@vip.163.com, Department of Neurology, Beijing Tiantan Hospital, Capital Medical University, Beijing, China.

Contributors TZ monitored document collection for the whole Guidelines, wrote the outline, drafted and revised the paper, and confirmed the level of evidence and classification. JZ wrote the motor rehabilitation of stroke, and drafted and revised the paper. XL wrote the part of complications in stroke rehabilitation. YB wrote the part of sensory dysfunction, dysarthria and cognitive impairment. BW wrote the part of mode of stroke rehabilitation. YQ wrote the part of mental disorder in stroke rehabilitation. BL wrote the swallowing dysfunction in stroke rehabilitation. SZ translated and revised the draft paper.

Funding This research received specific funding from Chinese Stroke Association Guidelines Writing Committee.

Competing interests None declared.

Patient consent for publication Not required.

Provenance and peer review Commissioned; externally peer reviewed.

Open access This is an open access article distributed in accordance with the Creative Commons Attribution Non Commercial (CC BY-NC 4.0) license, which permits others to distribute, remix, adapt, build upon this work non-commercially, and license their derivative works on different terms, provided the original work is properly cited, appropriate credit is given, any changes made indicated, and the use is non-commercial. See: http://creativecommons.org/licenses/by-nc/4.0/.

ORCID iD

Tong Zhang http://orcid.org/0000-0003-3467-6845

\section{REFERENCES}

1 Zhang T, Neurorehabilitation group, Society of Neurology, Chinese Medical Association. Guidelines for stroke rehabilitation in China. Chin J Rehabilitation Theory Pract 2012;04:301-18.

2 Nine Five research group. Study on early rehabilitation of acute stroke. Chin J Rehabil Med 2001;05:266-72.

3 Powers WJ, Rabinstein AA, Ackerson T, et al. 2018 guidelines for the early management of patients with acute ischemic stroke: a guideline 
for healthcare professionals from the American Heart Association/ American Stroke Association. Stroke 2018;49:e46-e110.

4 Maeshima S, Osawa A, Miyazaki Y, et al. Influence of dysphagia on short-term outcome in patients with acute stroke. Am J Phys Med Rehabil 2011;90:316-20.

5 McMicken BL, Muzzy CL. Functional outcomes of standard dysphagia treatment in first time documented stroke patients. Disabil Rehabil 2009;31:806-17.

6 Kendall KA, Ellerston J, Heller A, et al. Objective measures of swallowing function applied to the dysphagia population: a one year experience. Dysphagia 2016;31:538-46.

7 Kertscher B, Speyer R, Palmieri M, et al. Bedside screening to detect oropharyngeal dysphagia in patients with neurological disorders: an updated systematic review. Dysphagia 2014;29:204-12.

8 Miles A, Zeng ISL, McLauchlan $\mathrm{H}$, et al. Cough reflex testing in dysphagia following stroke: a randomized controlled trial. J Clin Med Res 2013;5:222-33.

9 Ikenaga $\mathrm{Y}$, Nakayama $\mathrm{S}$, Taniguchi $\mathrm{H}$, et al. Factors predicting recovery of oral intake in stroke survivors with dysphagia in a convalescent rehabilitation ward. J Stroke Cerebrovasc Dis 2017;26:1013-9.

10 Guillén-Solà A, Marco E, Martínez-Orfila J, et al. Usefulness of the volume-viscosity swallow test for screening dysphagia in subacute stroke patients in rehabilitation income. NeuroRehabilitation 2013;33:631-8.

11 Falsetti P, Acciai C, Palilla R, et al. Oropharyngeal dysphagia after stroke: incidence, diagnosis, and clinical predictors in patients admitted to a neurorehabilitation unit. J Stroke Cerebrovasc Dis 2009:18:329-35.

12 Flowers HL, Silver FL, Fang J, et al. The incidence, co-occurrence, and predictors of dysphagia, dysarthria, and aphasia after first-ever acute ischemic stroke. J Commun Disord 2013;46:238-48.

13 Brady MC, Clark AM, Dickson S, et al. The impact of stroke-related dysarthria on social participation and implications for rehabilitation. Disabil Rehabil 2011;33:178-86

14 Nobis-Bosch R, Springer L, Radermacher I, et al. Supervised home training of dialogue skills in chronic aphasia: a randomized parallel group study. J Speech Lang Hear Res 2011;54:1118-36.

15 Meltzer JA, Baird AJ, Steele RD, et al. Computer-based treatment of poststroke language disorders: a non-inferiority study of telerehabilitation compared to in-person service delivery. Aphasiology 2018;32:290-311.

16 Choi Y-H, Park HK, Ahn K-hwan, et al. A telescreening tool to detect aphasia in patients with stroke. Telemed J E Health 2015;21:729-34.

17 Zhou DHD, Wang JYJ, Li J, et al. Study on frequency and predictors of dementia after ischemic stroke: the Chongqing stroke study. $J$ Neurol 2004;251:421-7.

18 Madureira S, Guerreiro M, Ferro JM. Dementia and cognitive impairment three months after stroke. Eur J Neurol 2001;8:621-7.

19 Wang Y. How to improve the application of cognitive impairment scale in clinic. Chin J Intern Med 2005;09:78-9.

20 Wang W, Wang L. Application of the Montreal cognitive assessment in screening patients with mild cognitive impairment. Chin J Intern Med 2007;05:414-6.

21 Delavaran $\mathrm{H}$, Jönsson $\mathrm{A}-\mathrm{C}$, Lövkvist $\mathrm{H}$, et al. Cognitive function in stroke survivors: a 10-year follow-up study. Acta Neurol Scand 2017;136:187-94.

22 Jacquin $A$, Binquet $C$, Rouaud O, et al. Post-stroke cognitive impairment: high prevalence and determining factors in a cohort of mild stroke. J Alzheimers Dis 2014;40:1029-38.

23 Fu C, Jin X, Chen B, et al. Comparison of the mini-mental state examination and Montreal cognitive assessment executive subtests in detecting post-stroke cognitive impairment. Geriatr Gerontol Int 2017:17:2329-35.

24 Shi D, Chen X, Li Z. Diagnostic test accuracy of the Montreal Cognitive Assessment in the detection of post-stroke cognitive impairment under different stages and cutoffs: a systematic review and meta-analysis. Neurol Sci 2018;39:705-16.
25 Smith AC, Saunders DH, Mead G. Cardiorespiratory fitness after stroke: a systematic review. Int J Stroke 2012;7:499-510.

26 Shephard RJ. Maximal oxygen intake and independence in old age. Br J Sports Med 2009;43:342-6.

27 Flaster M, Sharma A, Rao M. Poststroke depression: a review emphasizing the role of prophylactic treatment and synergy with treatment for motor recovery. Top Stroke Rehabil 2013;20:139-50.

28 Hoffmann T, Ownsworth T, Eames S, et al. Evaluation of brief interventions for managing depression and anxiety symptoms during early discharge period after stroke: a pilot randomized controlled trial. Top Stroke Rehabil 2015;22:116-26.

29 Zhao F-Y, Yue Y-Y, Li L, et al. Clinical practice guidelines for poststroke depression in China. Braz J Psychiatry 2018;40:325-34.

30 Berg A, Lönnqvist J, Palomäki H, et al. Assessment of depression after stroke: a comparison of different screening instruments. Stroke 2009;40:523-9.

31 Maaijwee NAMM, Rutten-Jacobs LCA, Arntz RM, et al. Long-term increased risk of unemployment after young stroke: a long-term follow-up study. Neurology 2014;83:1132-8.

32 Kwah LK, Harvey LA, Diong JHL, et al. Half of the adults who present to hospital with stroke develop at least one contracture within six months: an observational study. J Physiother 2012;58:41-7.

33 Gillard PJ, Sucharew H, Kleindorfer D, et al. The negative impact of spasticity on the health-related quality of life of stroke survivors: a longitudinal cohort study. Health Qual Life Outcomes 2015;13:159.

34 Coggrave M, Norton C, Cody JD. Management of faecal incontinence and constipation in adults with central neurological diseases. Cochrane Database Syst Rev 2014:1:Cd002115.

35 Pizzi A, Falsini C, Martini M, et al. Urinary incontinence after ischemic stroke: clinical and urodynamic studies. Neurourol Urodyn 2014;33:420-5

36 Ersoz M, Erhan B, Akkoc Y, et al. An evaluation of bladder emptying methods and the effect of demographic and clinical factors on spontaneous voiding frequency in stroke patients. Neurol Sci 2013;34:729-34

37 Zhang K, Tang Q, Zhao C. Traditional manual acupuncture combined with rehabilitation therapy for shoulder hand syndrome after stroke within the Chinese healthcare system. Clin Rehabil 2019;33:1699-700.

38 Liu S, Zhang CS, Cai Y, et al. Acupuncture for post-stroke shoulderhand syndrome: a systematic review and meta-analysis. Front Neurol 2019;10:433.

39 Eun Young H, Hyeyun K, Sang Hee I, Hee S I. Pamidronate effect compared with a steroid on complex regional pain syndrome type I: pilot randomised trial. Neth J Med 2016;74:30-5.

40 Gritsch S, Bali KK, Kuner R, et al. Functional characterization of a mouse model for central post-stroke pain. Mol Pain 2016;12. doi:10.1177/1744806916629049. [Epub ahead of print: 08 Mar 2016]

41 Klit H, Finnerup NB, Jensen TS. Central post-stroke pain: clinical characteristics, pathophysiology, and management. Lancet Neurol 2009;8:857-68

42 Gouni-Berthold I, Berthold HK, Vitamin D. Vitamin D and vascular disease. Curr Vasc Pharmacol 2020. doi:10.2174/1570161118666200 317151955. [Epub ahead of print: 17 Mar 2020].

43 Bahat G, Ozkok S, Ozturk S, et al. Some comments for better understanding of the study entitled "Reduced vitamin D levels are associated with stroke-associated pneumonia in patients with acute ischemic stroke" [letter]. Clin Interv Aging 2020;15:159-60.

44 Kerse N, Parag V, Feigin VL, et al. Falls after stroke: results from the Auckland Regional Community Stroke (ARCOS) Study, 2002 to 2003. Stroke 2008;39:1890-3.

45 Huang $\mathrm{Y}$, Wu C, Peng $\mathrm{H}$, et al. The correlation between fall prevention knowledge and behavior in stroke outpatients. J Neurosci Nurs 2020;52:61-5.

46 Wei WE, De Silva DA, Chang HM, et al. Post-stroke patients with moderate function have the greatest risk of falls: a national cohort study. BMC Geriatr 2019;19:373. 